УДК 517.9

\title{
Nonlinear Related System of Inverted Pentulums Control
}

\author{
Mikhail E. Semenova, \\ Mikhail A. Popov ${ }^{\mathrm{b}}$ and Olesya I. Kanishcheva* \\ ${ }^{a}$ Military Education and Research Centre of Military-Air Forces \\ «Military-Air Academy \\ Named After Professor N.E. Zhukovsky and Yu.A. Gagarin» \\ 54a Starykh Bol'shevikov Str., Voronezh, 394064, Russia \\ ${ }^{b}$ Voronezh State Technical University \\ 8420 let Oktyabrya Str., Voronezh, 394006, Russia
}

Received 05.12.2017, received in revised form 04.01.2018, accepted 21.04.2018

In this paper we propose a linear and nonlinear mathematical model of linked inverse pendulums. We investigate dynamics of this mechanical system and determined the stability parameters. After that we presented results of experiments for various system configurations. In conclusion we constructed stability zones in the parameter space for linear and nonlinear systems. The system is controlled by feedback. The introduced nonlinear spring stiffness is part of the dynamic control. There are three stationary points in the phase space, however, only one of them has real coordinates. As a result of the study, it was shown that a complex unstable system consisting of oscillators with a nonlinear coupling can be described by a fairly simple system of equations, and its stabilization, under certain conditions, is possible with the help of a fairly simple control of the periodic feedback function.

Keywords: inverted pendulum, linked oscillators, stabilization, controlling.

Citation: Semenov M.E., Popov M.A., Kanishcheva O.I. Nonlinear related system of inverted pentulums control, J. Sib. Fed. Univ. Eng. technol., 2018, 11(3), 280-290. DOI: 10.17516/1999-494X-0040.

(C) Siberian Federal University. All rights reserved

* Corresponding author E-mail address: mk1150@mail.ru, soeltic@gmail.com,oleka_olesya@mail.ru 


\title{
Управление системой нелинейно связанных перевернутых маятников
}

\author{
М.Е. Семёнов ${ }^{\text {, }, ~ М . А . ~ П о п о в ~}{ }^{\boldsymbol{0}}$, О.И. Канищева ${ }^{\mathbf{a}}$ \\ ${ }^{a}$ Военный учебно-научный изентр Военно-воздушных сил \\ «Военно-воздушная академия \\ имени профессора Н.Е. Жуковского и Ю.А. Гагарина» \\ Россия, 371600, Воронеж, ул. Старых Большевиков, 54 а \\ ${ }^{6}$ Воронежский государственный технический университет \\ Россия, 394064, Воронеж, ул. 20-лет Октября, 84
}

\begin{abstract}
Предложена математическая модель связанных обратных маятников в линейной и нелинейной постановке. Проведено исследование динамики этой механической системы и определены предельные параметры, обеспечивающие возможность стабилизации. Приведеньь результаты экспериментов для различных конфигураций системы. Построены зоны устойчивости в пространстве параметров в линейной и нелинейной постановке. Управление системой осуществляется по обратной связи. Введенная нелинейная жесткость пружинь является частью динамического управления. При этом в фазовом пространстве существуют три стационарные точки, однако вещественные координаты имеет лишь одна из них. В результате проведенного исследования показано, что сложная неустойчивая система, состоящая из осиилляторов с нелинейной связью, может быть описана достаточно простой системой уравнений, а ее стабилизация при соблюдении определенных условий возможна с помощью достаточно простого управления периодической функичей по обратной связи.
\end{abstract}

Ключевые слова: обратный маятник, связанные осцилляторы, стабилизация, управление.

\section{Введение}

Теория колебаний нелинейных систем широко применяется при моделировании различных физических явлений: колебания молекул в жидкостях и твердых телах, электрических цепях, состоящих из нескольких взаимосвязанных контуров и т.д. [1] В таких системах реализуются разнообразные дисперсионные зависимости, на основе которых исследуется распространение волн в нелинейных средах. Подавляющее большинство моделей таких систем основывается на законах движения простейших связанных осцилляторов и их цепочек, динамика которых формализуется посредством как линейных, так и нелинейных уравнений. В большинстве подобных задач рассматриваются колебания маятников, у которых нулевое положение является устойчивым [2-4]. Подробный обзор последних результатов в этой области приведен, например, в [5].

В то же время в ряде практически важных задач (например, колебания атомов в телах с большой температурой, проблема стабилизации плазмы) нулевое положение неустойчиво. В связи с этим отметим классическую задачу стабилизации верхнего положения обратного маятника [6]. При решении этой задачи основное внимание уделяется проблеме стабилизации неустойчивого положения равновесия маятника путем движений нижней точки крепления. Этой проблеме посвящено огромное количество публикаций, достаточно подробный обзор которых содержится в [7]. Особенностью жесткого маятника с осциллирующим креплением является возможность динамической стабилизации его верхнего положения. Задача стабилизации ма-

$$
-281-
$$


ятника с помощью вертикальных осцилляций точки крепления хорошо изучена и сводится к уравнению Матье [8]. Теоретическое объяснение этого явления было сделано Стефенсоном в 1908 г. [9]. Физическое объяснение динамической стабилизации перевернутого маятника вертикальными осцилляциями точки крепления (с помощью управления по обратной связи) было предложено академиком П.Л. Капицей в 1951 г., выполнившим детальное экспериментальное исследование этого явления [10]. Также отметим работы, в которых стабилизация верхнего неустойчивого положения осуществлялась посредством гистерезисного управления [11-14].

\section{Постановка задачи}

В настоящей работе рассматривается система, состоящая из двух перевернутых маятников массами $m_{l}$ и $m_{2}$, связанных между собой пружиной с жесткостью $k$. Основная задача работы заключается в формировании такого закона управления горизонтальным движением точки крепления, при котором фазовые координаты системы остаются в ограниченной области фазового пространства. Иными словами, речь идет об условиях, накладываемых на параметры системы и управление, обеспечивающих диссипативность системы. Рассмотрим решение данной задачи для различных вариантов управления рассматриваемой системой (управление может быть приложено как к одному маятнику системы, так и к системе в целом) и в случае линейной и нелинейной жесткости системы.

\section{Случай управления одним маятником}

Рассмотрим систему с линейной жесткостью и управлением, приложенным к одному из маятников. Ее физическая модель представлена на рис. 1.

Задачу стабилизации будем решать в предположении, что тележка не имеет массы и движется без трения, а управляющее воздействие сообщает ей ускорение $u$. В этом случае уравнения динамики системы имеют вид

$$
\left\{\begin{array}{l}
J_{1} \ddot{\varphi}_{1}=m_{1} g l_{1} \sin \varphi_{1}+k h^{2}\left(\sin \varphi_{2}-\sin \varphi_{1}\right)-u \\
J_{2} \ddot{\varphi}_{2}=m_{2} g l_{2} \sin \varphi_{2}-k h^{2}\left(\sin \varphi_{2}-\sin \varphi_{1}\right)
\end{array},\right.
$$

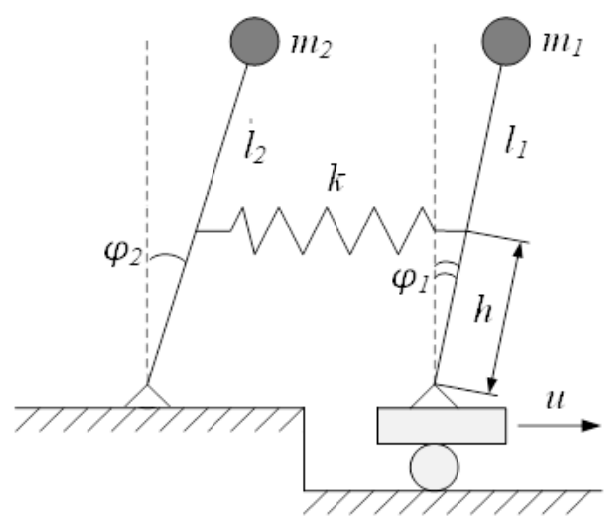

Рис. 1. Физическая модель связанных маятников

Fig. 1. Physical model of coupled pendulums 
где $\varphi_{1}, \varphi_{2}$ - углы отклонения маятников от вертикального положения. Предполагая отклонения от нулевого положения малыми, линеаризуем уравнения динамики. Тогда уравнения (1) примут вид

$$
\left\{\begin{array}{l}
\ddot{\varphi}_{1}=\left(\frac{m_{1} g l_{1}}{J_{1}}-\frac{k h^{2}}{J_{1}}\right) \varphi_{1}+\frac{k h^{2}}{J_{1}} \varphi_{2}-\frac{m_{1} l_{1}^{2}}{J_{1}} \ddot{y} \\
\ddot{\varphi}_{2}=\left(\frac{m_{2} g l_{2}}{J_{2}}-\frac{k h^{2}}{J_{2}}\right) \varphi_{2}+\frac{k h^{2}}{J_{2}} \varphi_{1}
\end{array}\right.
$$

Для дальнейшего рассмотрения систему (2) удобно представить в форме четырех уравнений первого порядка. Введем переменные: $z_{1}=\varphi_{1}, z_{2}=\dot{\varphi}_{1}, z_{3}=\varphi_{2}, \quad z_{4}=\dot{\varphi}_{2}$ и обозначим $B=\frac{k h^{2}}{J}, A=\frac{m g l}{J}-B$. С учетом введенных обозначений динамика системы будет описываться следующими уравнениями:

$$
\left\{\begin{array}{l}
\dot{z}_{1}=z_{2} \\
\dot{z}_{2}=A_{1} z_{1}+B_{1} z_{3}+u \\
\dot{z}_{3}=z_{4} \\
\dot{z}_{4}=B_{2} z_{1}+A_{2} z_{3}
\end{array}\right.
$$

или, в матричной форме,

$\Omega=\left(\begin{array}{cccc}0 & 1 & 0 & 0 \\ A_{1} & 0 & B_{1} & 0 \\ 0 & 0 & 0 & 1 \\ B_{2} & 0 & A_{2} & 0\end{array}\right), b=\left(\begin{array}{l}0 \\ 1 \\ 0 \\ 0\end{array}\right)$. Сформируем алгоритм управления по принципу обратной связи: $u=-D \cdot \frac{2}{\pi} \cdot \arctan (z \times c)$, где $D-$ параметр, характеризующий «интенсивность» управления. Отметим, что при отсутствии управления $(D=0)$ поведение системы будет определяться собственными числами матрицы системы. Эта матрица имеет четыре собственных числа: $\lambda_{1,2}= \pm \sqrt{\frac{A_{1}+A_{2}+\sqrt{\left(A_{1}-A_{2}\right)^{2}+4 B_{1} B_{2}}}{2}}, \lambda_{3,4}= \pm \sqrt{\frac{A_{1}+A_{2}-\sqrt{\left(A_{1}-A_{2}\right)^{2}+4 B_{1} B_{2}}}{2}}$. Два из них вещественные (одно положительно, второе отрицательно), а два других - чисто мнимые. Очевидно, что фазовое пространство делится на два подпространства. Колебания в одном из них (которое соответствует комплексным собственным числам) будут соответствовать особой точке центтр, а во втором подпространстве особая точка - седло [8].

Таким образом, задача стабилизации нулевого положения, соответствующего вертикальному положению обоих маятников, заключается в подавлении эффекта положительного собственного числа [14]. Такая система детально описана в работе [15], в которой было показано, что при достаточно большом значении параметра $D$ система (2) стабилизируема, - т.е. фазовые координаты остаются в ограниченной области фазового пространства. В настоящей рабо- 
те рассматривается пружина с нелинейной жесткостью, а именно предполагается изменение жесткости по закону

$$
k=k_{0}\left(1+x^{2}\right),
$$

где $x=h\left(\varphi_{1}-\varphi_{2}\right)$ или, во введенных выше обозначениях, $x=h\left(z_{1}-z_{3}\right)$. Таким образом, жесткость пружины, зависящая от взаимных отклонений маятников, является частью динамического управления. Перепишем систему (2), подставив в нее (5):

$$
\left\{\begin{array}{l}
\dot{z}_{1}=z_{2} \\
\dot{z}_{2}=\left(\frac{m_{1} g l_{1}}{J_{1}}-\frac{k_{0}\left(1+\left(h\left(z_{1}-z_{3}\right)\right)^{2}\right) h^{2}}{J_{1}}\right) z_{1}+\frac{k_{0}\left(1+\left(h\left(z_{1}-z_{3}\right)\right)^{2}\right) h^{2}}{J_{1}} z_{3}+u \\
\dot{z}_{3}=z_{4} \\
\dot{z}_{4}=\frac{k_{0}\left(1+\left(h\left(z_{1}-z_{3}\right)\right)^{2}\right) h^{2}}{J_{2}} z_{1}+\left(\frac{m_{2} g l_{2}}{J_{2}}-\frac{k_{0}\left(1+\left(h\left(z_{1}-z_{3}\right)\right)^{2}\right) h^{2}}{J_{2}}\right) z_{3}
\end{array} .\right.
$$

Сделаем замену переменных $\frac{m g l}{J}=\alpha, \frac{k_{0} h^{2}}{J}=\beta$. Тогда система (6) примет вид

$$
\left\{\begin{array}{l}
\dot{z}_{1}=z_{2} \\
\dot{z}_{2}=\alpha_{1} z_{1}-\beta_{1}\left(z_{1}-z_{3}\right)-\beta_{1} h^{2}\left(z_{1}-z_{3}\right)^{3}+u \\
\dot{z}_{3}=z_{4} \\
\dot{z}_{4}=\alpha_{2} z_{3}+\beta_{2}\left(z_{1}-z_{3}\right)+\beta_{2} h^{2}\left(z_{1}-z_{3}\right)^{3}
\end{array} .\right.
$$

Как известно, динамика любой системы связана с ее поведением в окрестности стационарных решений [16]. В рассматриваемом случае стационарные точки определяются как решения следующей системы алгебраических уравнений:

$$
\left.\begin{array}{rl}
z_{2} & =0 \\
\alpha_{1} z_{1}-\beta_{1}\left(z_{1}-z_{3}\right)-\beta_{1} h^{2}\left(z_{1}-z_{3}\right)^{3}+u=0 \\
z_{4}=0 \\
\alpha_{2} z_{3}+\beta_{2}\left(z_{1}-z_{3}\right)+\beta_{2} h^{2}\left(z_{1}-z_{3}\right)^{3}=0
\end{array}\right\} .
$$

Тогда в фазовом пространстве существует три точки равновесия, из которых вещественные координаты имеет только одна:

$$
P_{1}:\left\{\begin{array}{l}
z_{1}=\frac{1}{6 \Gamma_{2}^{3} h^{2}}\left(-6 \Gamma_{2}^{2} h^{2} \beta_{2} u-\frac{2 \sqrt[3]{18} \alpha_{2}^{2} \beta_{1}^{2} h^{2} \Gamma_{1} \Gamma_{2}^{3}}{\Gamma_{3}}+\sqrt[3]{12} \Gamma_{3}\right) \\
z_{2}=0 \\
z_{3}=\frac{\alpha_{1} \alpha_{2} \beta_{1} \beta_{2} h^{2}\left(2 \sqrt[3]{18} \Gamma_{1}^{3}-6 u \Gamma_{2}^{2} \Gamma_{3}-\alpha_{2}^{5} \beta_{1}^{5}\right)-\sqrt[3]{12} \alpha_{1} \beta_{2} \Gamma_{3}^{2}}{6 \alpha_{2} \beta_{1} h^{2} \Gamma_{2}^{3} \Gamma_{3}} \\
z_{4}=0
\end{array}\right\},
$$


где

$$
\begin{aligned}
& \Gamma_{1}=\alpha_{1} \alpha_{2}-\alpha_{1} \beta_{2}-\alpha_{2} \beta_{1}, \Gamma_{2}=\alpha_{2} b_{1}+\alpha_{1} b_{2}, \\
& \Gamma_{3}=\sqrt[3]{9 \alpha_{2}^{4} \beta_{1}^{3} h^{4} u \Gamma_{2}^{5}+\sqrt{3 \alpha_{2}^{6} \beta_{1}^{6} h^{6} \Gamma_{2}^{9}\left(4 \Gamma_{1}^{3}+27 \alpha_{2}^{2} h^{2} u^{2} \Gamma_{2}\right)}} .
\end{aligned}
$$

Исследуем устойчивость этого единственного стационарного решения. Для этого применим первый метод Ляпунова [17] по линейному приближению. Линеаризуем исходную систему в окрестности стационарного решения и найдем собственные числа матрицы линеаризованной системы:

$$
\operatorname{det}(\Theta-\lambda E)=0
$$

где $\Theta=\frac{\partial F}{\partial z}, z=\left(z_{1}, z_{2}, z_{3}, z_{4}\right)$. В рассматриваемом случае матрица линеаризованной системы будет иметь вид

$$
\Theta=\left|\begin{array}{cccc}
0 & 1 & 0 & 0 \\
\alpha_{1}-\beta_{1} \mu+c_{1} \chi & c_{2} \chi & \beta_{1} \mu+c_{3} \chi & c_{4} \chi \\
0 & 0 & 0 & 1 \\
\beta_{2} \mu & 0 & \alpha_{2}-\beta_{2} \mu & 0
\end{array}\right|,
$$

где $\mu=1+3 h^{2}\left(\bar{z}_{1}-\bar{z}_{3}\right)^{2}, \chi=\frac{-2 d}{\pi\left(1+(z \times c)^{2}\right)}, \bar{z}_{1}$ и $\bar{z}_{3}$ определяются соотношением $P_{1}$. Характеристическое уравнение (8) можно переписать в следующем виде:

$$
\lambda^{4}+\gamma_{1} \lambda^{3}+\gamma_{2} \lambda^{2}+\gamma_{3} \lambda+\gamma_{4}=0
$$

Подставим вычисленные ранее координаты точки $P_{1}$ и найдем собственные значения $\lambda_{1,2,3,4}$. Во избежание громоздких выкладок дальнейшие вычисления приведем для случая, в котором: величина управления $D=10$; начальная жесткость пружины $k_{0}=14.5$; высота крепления пружины $h=0.1$; длины и массы осцилляторов $l_{1}=0.1, l_{2}=0.15, m_{1}=0.1, m_{2}=0.2$. Получим собственные значения в точке $P_{1}: \lambda_{1}=-1.0127+i 0.7334, \lambda_{2}=-1.0127-i 0.7334, \lambda_{3}=-0.6582, \lambda_{4}=-0.2453$. Поскольку все показатели $\Lambda<0$, то при данных параметрах система асимптотически устойчива [16].

Динамика системы при значениях параметров, описанных выше, и начальных условиях: $z_{1}[0]=-0.001, z_{2}[0]=-0.001, z_{3}[0]=0.002, z_{4}[0]=-0.002$, изображена на рис. 2,3 .

Как видим, стационарное решение, определяемое координатами точки $P_{1}$, устойчиво по Ляпунову. Исследуем зависимость устойчивости стационарного решения от параметров $k_{0}$ и $D$. Для этого зададим диапазоны изменения интенсивности управления $D \in[1 ; 50]$ и параметра жесткости пружины $k_{0} \in[1 ; 20]$, а значения остальных параметров зафиксируем. Как известно из [16], стационарное решение устойчиво, если старший показатель Ляпунова отрицателен. Т.е. область устойчивости в пространстве параметров $k_{0}, D$ определяется как решение неравенства $\Lambda=\Lambda_{\max }\left(k_{0}, D\right)<0$. Результаты представлены на рис. 4 .

На графике рис. 4 отмечены области устойчивости системы в пространстве параметров. В областях, помеченных синим цветом, показатель Ляпунова $\Lambda>0$ - это значит, что система неустойчива. В белых же областях, наоборот, система асимптотически устойчива.

$$
-285-
$$



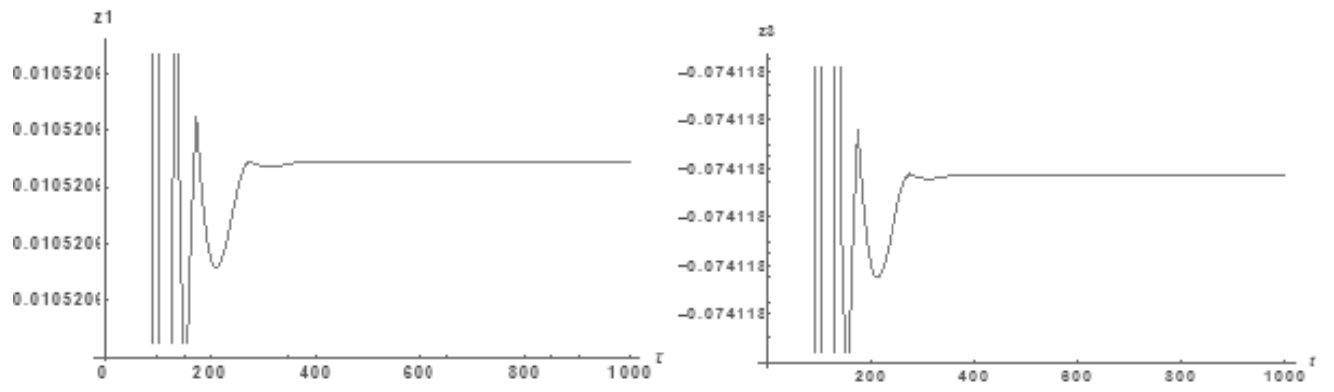

Рис. 2. Графики координат: $z_{1}$ (слева) и $z_{3}$ (справа)

Fig. 2. Velocity curves: $z_{1}$ (left) and $z_{3}$ (right)
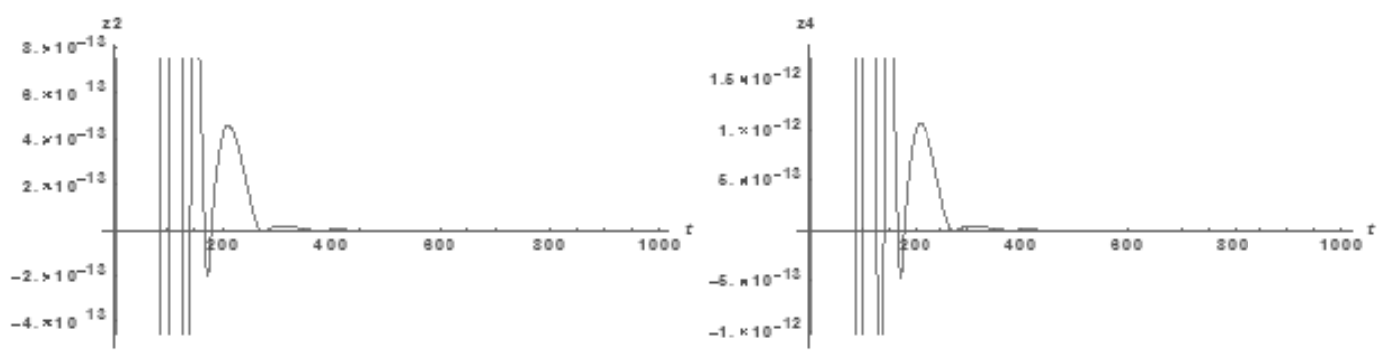

Рис. 3. Графики скоростей: $z_{2}$ (слева) и $z_{4}$ (справа)

Fig. 3. Speed graphs: $z_{2}$ (left) and $z_{4}$ (right)

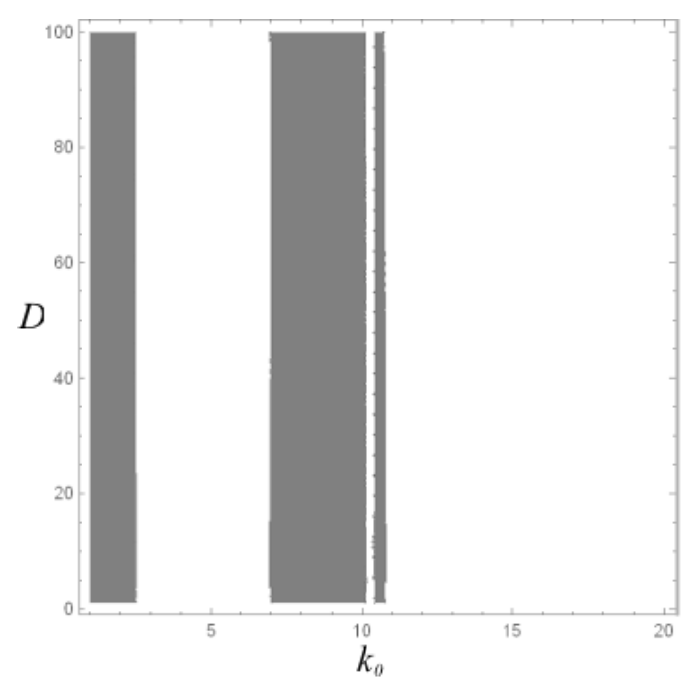

Рис. 4. Распределение значений $\operatorname{Re}[\max (\lambda)]$ в зависимости от значений $k_{0}$ и $D$

Fig. 4. Values distribution of $\operatorname{Re}[\max (\lambda)]$ in terms of values of $k_{0}$ and $D$ 


\section{Случай управления обоими маятниками}

Рассмотрим систему с нелинейной жесткостью и управлением, приложенным к обоим маятникам. Ее физическая модель представлена на рис. 5.

В данном случае в четвертом уравнении системы (4) добавится переменная управления $u$. Как и в предыдущем случае, имеется только одна стационарная точка:

$$
P_{1}:\left\{\begin{array}{l}
z_{1}=\frac{1}{18 \Gamma_{2}^{3} h^{2}}\left(\frac{6 \sqrt[3]{12} \alpha_{2}^{2} \beta_{1} h^{2} \Gamma_{1} \Gamma_{2}^{3}}{\Gamma_{3}}+\sqrt[3]{12} \Gamma_{3}\right) \\
z_{2}=0 \\
z_{3}=\frac{\alpha_{1} \alpha_{2} \beta_{1} \beta_{2} h^{2}\left(3 \sqrt[3]{18} \Gamma_{1}^{3}-6 u \Gamma_{2}^{2} \Gamma_{3}\right)-\sqrt[3]{18} \alpha_{1} \Gamma_{3}}{18 \alpha_{2} h^{2} \Gamma_{2}^{3} \Gamma_{3}} \\
z_{4}=0
\end{array}\right\} .
$$

Матрица линеаризованной системы имеет вид

$$
\Theta=\left|\begin{array}{cccc}
0 & 1 & 0 & 0 \\
\alpha_{1}-\beta_{1} \mu+c_{1} \chi & c_{2} \chi & \beta_{1} \mu+c_{3} \chi & c_{4} \chi \\
0 & 0 & 0 & 1 \\
\beta_{2} \mu+c_{1} \chi & c_{2} \chi & \alpha_{2}-\beta_{2} \mu+c_{3} \chi & c_{4} \chi
\end{array}\right|,
$$

где $\mu=1+3 h^{2}\left(\bar{z}_{1}-\bar{z}_{3}\right)^{2}, \chi=\frac{-2 d}{\pi\left(1+(z \times c)^{2}\right)}, \bar{z}_{1}$ и $\bar{z}_{3}$ определяются соотношением $P_{1}$. Характеристическое уравнение эквивалентно полученному ранее

$$
\left(\lambda^{4}+\gamma_{1} \lambda^{3}+\gamma_{2} \lambda^{2}+\gamma_{3} \lambda+\gamma_{4}=0\right) .
$$

Найдем собственные значения $\lambda_{1,2,3,4}$. Во избежание громоздких выкладок, дальнейшие вычисления приведем для случая, в котором начальная жесткость пружины $k_{0}=100$, остальные параметры такие же, как и в предыдущем случае. Получим собственные значения: $\lambda_{1}=-0.8794+i 10.9709, \lambda_{2}=-0.8794-i 10.9709, \lambda_{3}=-0.5705, \lambda_{4}=-0.3231$. Тогда показатели

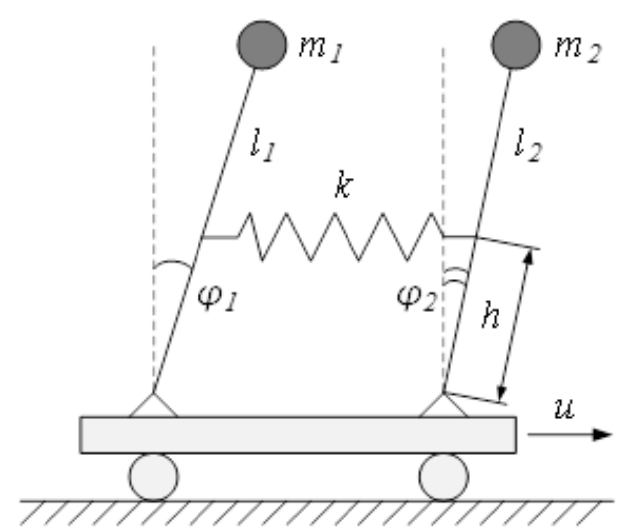

Рис. 5. Физическая модель связанных маятников

Fig. 5. Coupled pendulums physical model 
Ляпунова: $\Lambda_{1}=\Lambda_{2}=\operatorname{Re} \lambda_{2}=-0.8794, \Lambda_{3}=\lambda_{3}=-0.5705, \Lambda_{4}=\lambda_{4}=-0.3231$. Поскольку все показатели $\Lambda<0$, то при данных выбранных параметрах система устойчива [16]. Результаты моделирования описанной системы представлены на рис. 6, 7.

Как видно из графиков (рис. 6-7), при моделировании с заданными начальными условиями система диссипативна. Исследуем зависимость устойчивости стационарного решения от параметров $k_{0}$ и $D$. Для этого зададим диапазоны изменения интенсивности управления $D \in[1 ; 50]$ и параметра жесткости пружины $k_{0} \in[1 ; 20]$, а значения остальных параметров зафиксируем. Результаты даны на рис. 8.

На графике рис. 8 отмечены области устойчивости системы в пространстве параметров. В областях, помеченных синим цветом, показатель Ляпунова $\Lambda>0$ - это значит, что система неустойчива. В белых же областях, наоборот, система устойчива.

\section{Заключение}

В результате проведенного исследования было показано, что сложная неустойчивая система, состоящая из осцилляторов с нелинейной связью, может быть описана достаточно простой системой уравнений, а ее стабилизация при соблюдении определенных условий возможна с помощью достаточно простого управления периодической функцией по обратной связи.
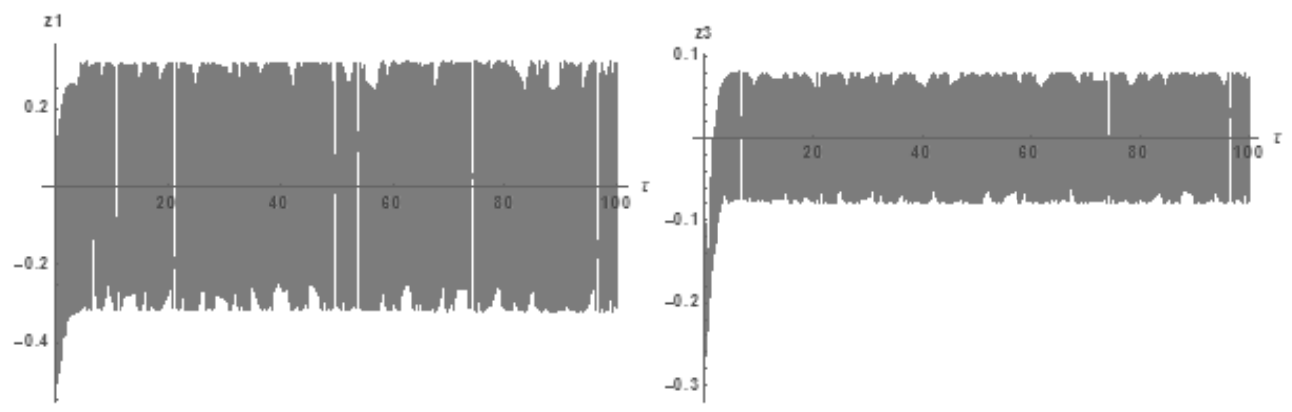

Рис. 6. Графики координат: $z_{1}$ (слева) и $z_{3}$ (справа)

Fig. 6. Coordinate graphs: $z_{1}$ (left) and $z_{3}$ (right)
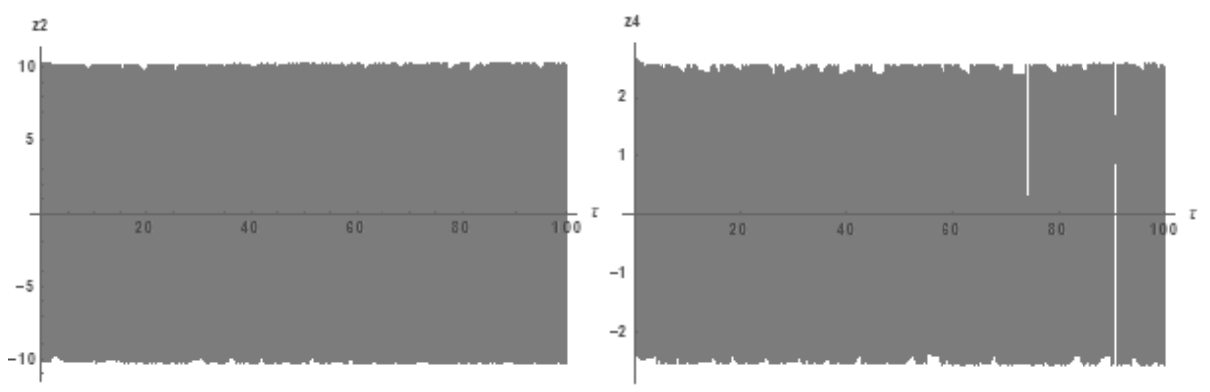

Рис. 7. Графики скоростей: $z_{2}$ (слева) и $z_{4}$ (справа)

Fig. 7. Velocity curves: $z_{2}$ (left) and $z_{4}$ (right) 


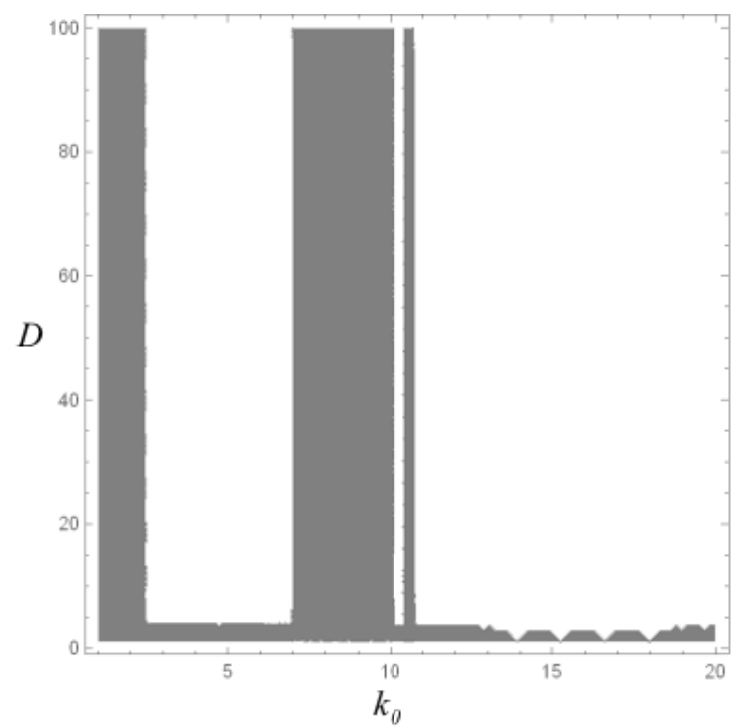

Рис. 8. Распределение значений $\operatorname{Re}[\max (\lambda)]$ в зависимости от значений $k_{0}$ и $D$

Fig. 8. Values distribution of $\operatorname{Re}[\max (\lambda)]$ in terms of values of $k_{0}$ and $D$

Работа выполнена при поддержке РФФИ, гранты № 17-01-00251, 16-08-00312.

\section{Список литературы}

[1] Андронов А.А., Витт А.А., Хайкин С.Э. Теория колебаний. М.: Наука, 1981. 568 с. [Andronov A.A., Witt A.A., Khaikin S.E. Theory of oscillations. Moscow, Nauka, 1981. 568 p. (in Russian)]

[2] Баутин Н.Н. О числе предельных циклов, появляющихся при изменении коэффициентов из состояния равновесия типа фокуса или центра, Математический сборник, 1952, 30(72), 181-196. [Bautin N.N. On the number of limit cycles appearing when the coefficients change from an equilibrium state of the focus or center type, Matematicheskii sbornik, 1952, 30(72), 181-196. (in Russian)]

[3] Трубецков Д.И., Рожнев А.Г. Линейные колебания и волны. М.: Издательство физикоматематической литературы, 2001. 416 с. [Trubetskov D.I., Rozhnev A.G. Linear oscillations and waves. Moscow, Publishing House of Physical and Mathematical Literature, 2001. 416 p. (in Russian)]

[4] Магнус К. Колебания: Введение в исследование колебательных систем. Пер. с нем. М.: Мир, 1982. 304 c. [Magnus K. Oscillations: Introduction to research of oscillatory systems. Translation from German. Moscow, Mir, 1982. 304 p. (in Russian)]

[5] Осипов Г.В. Синхронизация в неоднородных сетях осиилляторов. Нижний Новгород, 2014. 135 c. [Osipov G.V. Synchronization in heterogeneous networks of oscillators. Nizhny Novgorod, 2014. 135 p. (in Russian)]

[6] Колмогоров А.Н. О сохранении условно периодических движений при малом изменении функции Гамильтона, Доклады АН СCCP, 1954, 98(4), 527-530. [Kolmogorov A.N. On the conservation of conditionally periodic motions for a small change in the Hamiltonian function, Doklady Akad. Nauk USSR, 1954, 98(4), 527-530. (in Russian)]

$$
-289-
$$


[7] Бутиков Е.И. Стабилизация перевернутого маятника (60 лет маятнику Капицы), Компьютерные инструменты в образовании, 2010, 5, 3951. [Butikov E.I. Stabilization of the inverted pendulum (60 years of Kapitza's pendulum), Computer tools in education, 2010, 5, 39-51. (in Russian)]

[8] Неймарк Ю.И., Коган Н.Я., Савельев В.П. Динамические модели теории управления. М.: Наука, 1985. 400 c. [Neimark Yu.I., Kogan N.Ya., Savelyev V.P. Dynamic models of control theory. Moscow, Nauka, 1985. 400 p. (in Russian)]

[9] Stephenson A. «On an induced stability», Phil. Mag, 1908, 15(233).

[10] Капица П.Л. Маятник с вибрирующим подвесом, УФН, 1951, 44, 720. [Kapitsa P.L. A pendulum with a vibrating suspension, $U F N, 1951,44,7-20$. (in Russian)]

[11] Матвеев М.Г., Семенов М.Е., Шевлякова Д.В., Канищева О.И. Зоны устойчивости и периодические решения перевернутого маятника с гистерезисным управлением, Мехатроника, Автоматизация, Управление, 2012, 11, 814. [Matveev M.G., Semenov M.E., Shevlyakova D.V., Kanishcheva O.I. Zone of Stability and Periodic Solutions of the Inverted Pendulum with Hysteretic Control, Mechatronics, Automation, Control, 2012, 11, 8-14. (in Russian)]

[12] Семенов М.Е., Хатиф 3., Решетова О.О., Демчук А.А., Мелешенко П.А. Модель динамики обратного маятника с гистерезисным управлением, Вестник Воронежского государственного университета. Серия: Физика. Математика, 2016, 4, 165-177. [Semenov M.E., Hatif Z., Reshetova O.O., Demchuk A.A., Meleshenko P.A. The model of the dynamics with the inverted pendulum hysteretic control, Proceedings of Voronezh State University. Series: Physics. Mathematics, 2016, 4, 165-177. (in Russian)]

[13] Семенов М.Е., Матвеев М.Г., Лебедев Г.Н., Соловьев А.М. Стабилизация обратного гибкого маятника с гистерезисными свойствами, Мехатроника, Автоматизация, Управление, 2017, 8, 516-525. [Semenov M.E., Matveev M.G., Lebedev G.N., Solovyev A.M. Stabilization of a Flexible Inverted Pendulum with the Hysteretic Properties, Mechatronics, Automation, Control, 2017, 8, 516-525 (in Russian)]

[14] Solovyov A.M., Semenov M.E., Meleshenko P.A., Reshetova O.O., Popov M.A., Kabulova E.G. Hysteretic nonlinearity and unbounded solutions in oscillating systems, Procedia Engineering, 2017, 201, 549-555.

[15] Семенов М.Е., Соловьев А.М., Попов М.А. Стабилизация неустойчивых объектов: связанные осцилляторы, Труды МАИ, 2017, 93. [Semenov M.E., Solovyov A.M., Popov M.A. Stabilization of unstable objects: coupled oscillators, Trudy MAI, 2017, 93 (in Russian)]

[16] Кузнецов С.П. Динамический хаос. М.: Физматлит, 2006. 356 c. [Kuznetsov S.P. Dynamic chaos. Moscow, Fizmatlit, 2006. 356 p. (in Russian)]

[17] Мирошник И.В. Теория автоматического управления. Нелинейные и оптимальные системы. СПб.: Питер, 2006. 272 с. [Miroshnik I.V. Theory of automatic control. Nonlinear and optimal systems. St. Petersburg, Piter, 2006. 272 p. (in Russian)] 